2011 Shanghai Asia-Pacific School and Workshop on Gravitation International Journal of Modern Physics: Conference Series Vol. 7 (2012) 227-236

(C) World Scientific Publishing Company

DOI: $10.1142 / \mathrm{S} 2010194512004308$

\title{
THE KERR-NEWMAN/CFTS CORRESPONDENCE
}

\author{
CHIANG-MEI CHEN \\ Department of Physics and Center for Mathematics and Theoretical Physics, \\ National Central University, \\ Chungli 320, Taiwan \\ cmchen@phy.ncu.edu.tw \\ JIA-RUI SUN \\ Department of Physics, National Central University, \\ Chungli 320, Taiwan \\ jrsun@phy.ncu.edu.tw
}

\begin{abstract}
In this article, we review recent studies on multiple dual 2D CFT descriptions of the Kerr-Newman black hole, in terms of the Kerr/CFT and Reissner-Nordström/CFT correspondences. A microscopic hair conjecture is suggested.
\end{abstract}

Keywords: AdS/CFT correspondence; quantum gravity; black holes.

PACS numbers:

\section{Introduction}

The near horizon geometries of certain extremal black holes contain the $\mathrm{AdS}_{2}$ or $\mathrm{AdS}_{3}$ structures, which are known to play important role in understanding the macroscopic and microscopic entropies of black holes long ago. The well known examples include the Bertotti-Robinson solution $\left(\mathrm{AdS}_{2}\right)$ for extremal ReissnerNordström (RN) black hole in Einstein-Maxwell theory ${ }^{1,2}$, and the near horizon geometry $\left(\mathrm{AdS}_{3}\right)$ of the D1-D5 system in string theory ${ }^{3}$. Based on the holographic principle $^{4,5}$ and its realization in the asymptotically AdS spacetime, one can relate the quantum gravity description of black holes with their dual CFT in terms of the AdS/CFT correspondence ${ }^{6,7,8}$. In the past three years, new advances have been made in studying the CFT descriptions of a class of extremal black holes in which the near horizon geometries are of the warped $\mathrm{AdS}_{3}$ structure (with $S L(2, R)_{R} \times U(1)_{L}$ isometry). The initial progress was the calculation of the left hand central charge and temperature of the 2D CFT dual to the extremal Kerr black hole, called the Kerr/CFT correspondence ${ }^{9}$. The Kerr/CFT duality was further generalized into the near extremal and nonextremal cases by comparing the scattering amplitudes of external probe fields from the gravity side with those of the corresponding operators in the dual 2D CFT side ${ }^{10,11}$. Especially, even though the nonextremal Kerr 
black hole doesn't contain the warped $\mathrm{AdS}_{3}$ geometry, a 2D hidden conformal symmetry can be detected by a probe scalar field at low frequencies. Other nonextremal black holes are shown to have the similar property, see ${ }^{12,13,14,15,16,17,18}$. There are various related studies and generalizations ${ }^{19,20,21,22,23,24,25,26,27,28,29,30,31,32}$, see a recent review ${ }^{33}$ for more references therein.

A nontrivial generalization of the Kerr/CFT correspondence is the studying of the CFT descriptions for nonrotating charged black holes, the simplest example is the (near) extremal 4D RN black hole, which only contains a near horizon $\mathrm{AdS}_{2}$ geometry, but accompanied by an $U(1)$ gauge field. The key observation was that the background $U(1)$ gauge field plays an equivalent role as that of the rotation in rotating black holes, consequently a warped $\mathrm{AdS}_{3}$ structure is formed together with the $\mathrm{AdS}_{2}$ base manifold. This fact enables us to investigate the (near) extremal RN/CFT duality $22,23,24,25$, and the 2D CFT description for nonextremal RN black hole ${ }^{12,17}$.

Combined with the results in the Kerr/CFT and RN/CFT correspondences, it is expectable that a multiple dual 2D CFT descriptions should exist for charged rotating black holes, e.g., the Kerr-Newman (KN) black hole. We showed that, besides the angular momentum $J$-picture (associated with the Kerr/CFT duality, where the central charges of the dual 2D CFT are $c_{L}=c_{R}=12 J$ ) revealed in ${ }^{13,16,22}$, another $Q$-picture (associated with the RN/CFT duality, in which the central charges are $c_{L}=c_{R}=6 Q^{3} / \ell$, where $\ell$ is the parameter depends on the embedding) does exist for the general nonextremal KN black hole by probing the 2D hidden conformal symmetries via an external charged scalar field ${ }^{18}$. Accordingly, a KN/CFTs correspondence was suggested. This suggestion was checked by matching of the absorption cross sections and real time correlators calculated in both the $J$ - and the $Q$-pictures from the gravity and the CFT sides. It is natural to believe that these two individual descriptions are just special limits of a unknown full holographic dual description of the KN black hole. The KN/CFTs duality could be much more easily understood in the geometric picture since in the near extremal limit, the near horizon KN black hole contains an $\mathrm{AdS}_{2}$ geometry together with two $U(1)$ fibers, with $S L(2, R)_{R} \times U(1) \times U(1)$ isometry. Thus there are two possible choices to form the desired warped $\mathrm{AdS}_{3}$ structures, and certainly to have two individual dual 2D CFT descriptions. A similar idea has been studied later for the spinning M5 branes in ${ }^{34}$. Moreover, our work also suggested a fascinating "microscopic hair conjecture" for each macroscopic hair parameter, in additional to the mass of a black hole in the Einstein-Maxwell theory (with dimension $D \geq 3$ ), there should exist an associated holographic $\mathrm{CFT}_{2}$ description.

This article is organized as follows. We first review some basic properties of the KN black hole and study the scattering of a probe charged scalar field in section II. In sections III and IV, we explicitly analyze the $J$-picture and the $Q$-picture of the KN black hole, respectively, including the probing of hidden conformal symmetry, the calculation of absorption cross sections and real time correlators. Then we give the conclusion and discussion in section $\mathrm{V}$. 


\section{Charged Scalar Field in the Kerr-Newman Background}

The 4D Kerr-Newman black hole in the Boyer-Lindquist coordinates is

$$
\begin{aligned}
d s^{2}= & -\frac{\Delta-a^{2} \sin ^{2} \theta}{\Sigma}\left[d t+\frac{\left(2 M r-Q^{2}\right) a \sin ^{2} \theta}{\Delta-a^{2} \sin ^{2} \theta} d \phi\right]^{2} \\
& +\Sigma\left(\frac{d r^{2}}{\Delta}+d \theta^{2}+\frac{\Delta \sin ^{2} \theta}{\Delta-a^{2} \sin ^{2} \theta} d \phi^{2}\right), \\
A_{[1]}= & -\frac{Q r}{\Sigma}\left(d t-a \sin ^{2} \theta d \phi\right),
\end{aligned}
$$

where the three macroscopic hairs are the mass $M$, electric charge $Q$ and angular momentum $J=M a$, and

$$
\Sigma=r^{2}+a^{2} \cos ^{2} \theta, \quad \Delta=r^{2}-2 M r+a^{2}+Q^{2} .
$$

The radii of black hole outer and inner horizons $r_{ \pm}$, the horizon angular velocity $\Omega_{H}$ and the chemical potential $\Phi_{H}$ are

$$
r_{ \pm}=M \pm \sqrt{M^{2}-a^{2}-Q^{2}}, \quad \Omega_{H}=\frac{a}{r_{+}^{2}+a^{2}}, \quad \Phi_{H}=\frac{Q r_{+}}{r_{+}^{2}+a^{2}} .
$$

In addition, the Hawking temperature and the black hole entropy, are

$$
T_{H}=\frac{\kappa}{2 \pi}=\frac{r_{+}-r_{-}}{4 \pi\left(r_{+}^{2}+a^{2}\right)}, \quad S_{B H}=\frac{A_{+}}{4}=\pi\left(r_{+}^{2}+a^{2}\right),
$$

where $\kappa$ and $A_{+}$are the surface gravity and area of the outer horizon, respectively.

The Klein-Gordon (KG) equation of a massive probe charged scalar field $\Phi$ scattering in the $\mathrm{KN}$ black hole is

$$
\left(\nabla_{\alpha}-i q A_{\alpha}\right)\left(\nabla^{\alpha}-i q A^{\alpha}\right) \Phi=\mu^{2} \Phi,
$$

where $\mu$ and $q$ are mass and electric charge parameters of the scalar field respectively. After assuming the following mode expansions

$$
\Phi(t, r, \theta, \phi)=\mathrm{e}^{-i \omega t+i m \phi} R(r) S(\theta),
$$

the $\mathrm{KG}$ equation can be decoupled into the angular and radial equations as

$$
\begin{array}{r}
\frac{1}{\sin \theta} \partial_{\theta}\left(\sin \theta \partial_{\theta} S\right)-\left[a^{2}\left(\omega^{2}-\mu^{2}\right) \sin ^{2} \theta+\frac{m^{2}}{\sin ^{2} \theta}-\lambda\right] S=0, \\
\partial_{r}\left(\Delta \partial_{r} R\right)+\left[\frac{\left[\left(r^{2}+a^{2}\right) \omega-q Q r-m a\right]^{2}}{\Delta}-\mu^{2}\left(r^{2}+a^{2}\right)+2 m a \omega-\lambda\right] R=0,
\end{array}
$$

where $\lambda$ is the separation constant. When $\mu=0$, the radial equation can be further expressed in a symmetric form

$$
\begin{aligned}
& \partial_{r}\left(\Delta \partial_{r} R\right)+\left[\frac{\left[\left(r_{+}^{2}+a^{2}\right) \omega-a m-Q r_{+} q\right]^{2}}{\left(r-r_{+}\right)\left(r_{+}-r_{-}\right)}-\frac{\left[\left(r_{-}^{2}+a^{2}\right) \omega-a m-Q r_{-} q\right]^{2}}{\left(r-r_{-}\right)\left(r_{+}-r_{-}\right)}\right] R \\
& \quad+\left[\omega^{2} r^{2}+2(\omega M-q Q) \omega r+\omega^{2} a^{2}-\omega^{2} Q^{2}+(2 \omega M-q Q)^{2}\right] R=\lambda R .
\end{aligned}
$$


Under conditions: (1) small frequency $\omega M \ll 1$ (consequently $\omega a \ll 1$ and $\omega Q \ll 1$ ), (2) small probe charge $q Q \ll 1$ and (3) near region $\omega r \ll 1$, the potential terms in the second line can be neglected, and $\lambda=\lambda_{l}=l(l+1)$. Then eq.(9) reduces to

$\partial_{r}\left(\Delta \partial_{r} R\right)+\left[\frac{\left[\left(r_{+}^{2}+a^{2}\right) \omega-a m-Q r_{+} q\right]^{2}}{\left(r-r_{+}\right)\left(r_{+}-r_{-}\right)}-\frac{\left[\left(r_{-}^{2}+a^{2}\right) \omega-a m-Q r_{-} q\right]^{2}}{\left(r-r_{-}\right)\left(r_{+}-r_{-}\right)}\right] R=\lambda_{l} R$.

In the following two sections, we will show how to probe the twofold $2 \mathrm{D}$ conformal symmetries hidden in eq.(10). We first reproduce the $J$-picture in terms of the Kerr/CFT correspondence studied in ${ }^{13,14}$, and then investigate the new $Q$-picture in terms of the RN/CFT correspondence. We just list the main results, for detailed calculation, please refer to ${ }^{18}$.

\section{Angular Momentum ( $J-)$ Picture}

\subsection{Hidden Conformal Symmetry}

To probe the $J$-picture $\mathrm{CFT}_{2}$ description dual to the $\mathrm{KN}$ black hole, one should consider a neutral scalar field by imposing $q=0$, then eq.(10) becomes

$$
\left(\partial_{r}\left(\Delta \partial_{r}\right)-\frac{\left[\left(r_{+}^{2}+a^{2}\right) \partial_{t}+a \partial_{\phi}\right]^{2}}{\left(r-r_{+}\right)\left(r_{+}-r_{-}\right)}+\frac{\left[\left(r_{-}^{2}+a^{2}\right) \partial_{t}+a \partial_{\phi}\right]^{2}}{\left(r-r_{-}\right)\left(r_{+}-r_{-}\right)}\right) \Phi=l(l+1) \Phi .
$$

It was shown that the left hand side of the above equation is just the $S L(2, R)$ Casimir operator acting on $\Phi$ with the identifications of parameters ${ }^{18}$

$$
T_{L}^{J}=\frac{r_{+}^{2}+r_{-}^{2}+2 a^{2}}{4 \pi a\left(r_{+}+r_{-}\right)}, \quad T_{R}^{J}=\frac{r_{+}-r_{-}}{4 \pi a}, \quad n_{L}^{J}=-\frac{1}{2\left(r_{+}+r_{-}\right)}, \quad n_{R}^{J}=0 .
$$

Since the neutral probe scalar field does not couple with the background gauge field, it only reveals the angular momentum dominated section of the dual field theory, resembling the 2D CFT dual to the Kerr black hole with central charges

$$
c_{L}^{J}=c_{R}^{J}=12 J=12 M a .
$$

One can directly check that the CFT microscopic entropy from the Cardy formula indeed reproduces the Bekenstein-Hawking area entropy of the KN black hole:

$$
S_{\mathrm{CFT}}^{J}=\frac{\pi^{2}}{3}\left(c_{L}^{J} T_{L}^{J}+c_{R}^{J} T_{R}^{J}\right)=\pi\left(r_{+}^{2}+a^{2}\right)=S_{\mathrm{BH}} .
$$

\subsection{Scattering}

The near region solutions of eq.(10) include ingoing and outgoing modes as

$$
\begin{aligned}
R_{J}^{(\text {in })} & =z^{-i \gamma_{J}}(1-z)^{l+1} F\left(a_{J}, b_{J} ; c_{J} ; z\right), \\
R_{J}^{\text {(out) }} & =z^{i \gamma_{J}}(1-z)^{l+1} F\left(a_{J}^{*}, b_{J}^{*} ; c_{J}^{*} ; z\right),
\end{aligned}
$$


where $q=0$ is imposed, $z=\frac{r-r_{+}}{r-r_{-}}$, the expressions of $a_{J}, b_{J}, c_{J}$ and $\gamma_{J}$ are

$$
\begin{aligned}
& \gamma_{J}=\frac{\omega\left(r_{+}^{2}+a^{2}\right)-m a}{r_{+}-r_{-}}, \\
& b_{J}=1+l-i \omega\left(r_{+}+r_{-}\right), \quad a_{J}=1+l-i \frac{\omega\left(r_{+}^{2}+r_{-}^{2}+2 a^{2}\right)-2 m a}{r_{+}-r_{-}},
\end{aligned}
$$

The asymptotic form of the ingoing mode is

$$
R_{J}^{(\mathrm{in})}(r \gg M) \sim A_{J} r^{l}+B_{J} r^{-l-1},
$$

where

$$
A_{J}=\frac{\Gamma\left(c_{J}\right) \Gamma(2 l+1)}{\Gamma\left(a_{J}\right) \Gamma\left(b_{J}\right)}, \quad B_{J}=\frac{\Gamma\left(c_{J}\right) \Gamma(-2 l-1)}{\Gamma\left(c_{J}-a_{J}\right) \Gamma\left(c_{J}-b_{J}\right)},
$$

and the conformal weights of the operator dual to the scalar field are

$$
h_{L}^{J}=h_{R}^{J}=l+1
$$

Hence, $a_{J}$ and $b_{J}$ can be expressed in terms of $\omega_{L}^{J}$ and $\omega_{R}^{J}$

$$
a_{J}=h_{R}^{J}-i \frac{\omega_{R}^{J}}{2 \pi T_{R}^{J}}, \quad b_{J}=h_{L}^{J}-i \frac{\omega_{L}^{J}}{2 \pi T_{L}^{J}},
$$

with

$$
\omega_{L}^{J}=\frac{\omega\left(r_{+}^{2}+r_{-}^{2}+2 a^{2}\right)}{2 a}, \quad \omega_{R}^{J}=\frac{\omega\left(r_{+}^{2}+r_{-}^{2}+2 a^{2}\right)-2 m a}{2 a} .
$$

The essential part of the absorption cross section can be estimated as

$$
P_{\mathrm{abs}}^{J} \sim\left|A_{J}\right|^{-2} \sim \sinh \left(2 \pi \gamma_{J}\right)\left|\Gamma\left(a_{J}\right)\right|^{2}\left|\Gamma\left(b_{J}\right)\right|^{2} .
$$

Further with the help of the first law of thermodynamics,

$$
\delta S_{C F T}^{J}=\frac{\delta E_{L}^{J}}{T_{L}^{J}}+\frac{\delta E_{R}^{J}}{T_{R}^{J}}=\delta S_{B H}=\frac{1}{T_{H}} \delta M-\frac{\Phi_{H}}{T_{H}} \delta Q-\frac{\Omega_{H}}{T_{H}} \delta J,
$$

the absorption cross section can be expressed as ${ }^{14}$

$$
\begin{aligned}
P_{\mathrm{abs}}^{J} \sim & \left(T_{L}^{J}\right)^{2 h_{L}^{J}-1}\left(T_{R}^{J}\right)^{2 h_{R}^{J}-1} \sinh \left(\frac{\omega_{L}^{J}}{2 T_{L}^{J}}+\frac{\omega_{R}^{J}}{2 T_{R}^{J}}\right) \\
& \times\left|\Gamma\left(h_{L}^{J}+i \frac{\omega_{L}^{J}}{2 \pi T_{L}^{J}}\right)\right|^{2}\left|\Gamma\left(h_{R}^{J}+i \frac{\omega_{R}^{J}}{2 \pi T_{R}^{J}}\right)\right|^{2},
\end{aligned}
$$

which is the finite temperature absorption cross section of an operator dual to the neutral probe scalar field in the $J$-picture 2D CFT dual to the KN black hole. 


\subsection{Real-time Correlator}

The two-point retarded correlator calculated from the gravity side is ${ }^{31,35}$

$$
\begin{aligned}
G_{R}^{J} \sim \frac{B_{J}}{A_{J}}= & (-)^{h_{L}^{J}+h_{R}^{J}} \sin \left(i \frac{\omega_{L}^{J}}{2 T_{L}^{J}}\right) \sin \left(i \frac{\omega_{R}^{J}}{2 T_{R}^{J}}\right) \\
& \times \Gamma\left(h_{L}^{J}-i \frac{\omega_{L}^{J}}{2 \pi T_{L}^{J}}\right) \Gamma\left(h_{L}^{J}+i \frac{\omega_{L}^{J}}{2 \pi T_{L}^{J}}\right) \\
& \times \Gamma\left(h_{R}^{J}-i \frac{\omega_{R}^{J}}{2 \pi T_{R}^{J}}\right) \Gamma\left(h_{R}^{J}+i \frac{\omega_{R}^{J}}{2 \pi T_{R}^{J}}\right) .
\end{aligned}
$$

From the CFT side, the retarded Green function $G_{R}\left(\omega_{L}, \omega_{R}\right)$ can be obtained from the Euclidean correlator (in terms of the Euclidean frequencies $\omega_{E L}=i \omega_{L}$, and $\left.\omega_{E R}=i \omega_{R}\right)$

$$
\begin{aligned}
G_{E}\left(\omega_{E L}, \omega_{E R}\right) \sim & T_{L}^{2 h_{L}-1} T_{R}^{2 h_{R}-1} \mathrm{e}^{i \frac{\tilde{\omega}_{E L}}{2 T_{L}}} \mathrm{e}^{i \frac{\tilde{\omega}_{E R}}{2 T_{R}}} \\
& \times \Gamma\left(h_{L}-\frac{\tilde{\omega}_{E L}}{2 \pi T_{L}}\right) \Gamma\left(h_{L}+\frac{\tilde{\omega}_{E L}}{2 \pi T_{L}}\right) \\
& \times \Gamma\left(h_{R}-\frac{\tilde{\omega}_{E R}}{2 \pi T_{R}}\right) \Gamma\left(h_{R}+\frac{\tilde{\omega}_{E R}}{2 \pi T_{R}}\right),
\end{aligned}
$$

(where $\tilde{\omega}_{E L}=\omega_{E L}-i q_{L} \mu_{L}$ and $\tilde{\omega}_{E R}=\omega_{E R}-i q_{R} \mu_{R}$ ) by the analytic continuation from the on the upper half complex $\omega_{L, R}$-plane

$$
G_{E}\left(\omega_{E L}, \omega_{E R}\right)=G_{R}\left(i \omega_{L}, i \omega_{R}\right), \quad \omega_{E L}, \omega_{E R}>0,
$$

and the Euclidean frequencies $\omega_{E L}$ and $\omega_{E R}$ should take discrete values of the Matsubara frequencies $\left(m_{L}, m_{R}\right.$ are integers for bosons and half integers for fermions)

$$
\omega_{E L}=2 \pi m_{L} T_{L}, \quad \omega_{E R}=2 \pi m_{R} T_{R} .
$$

At these frequencies, the retarded Green function precisely agrees with the gravity side computation eq.(25) up to a numerical normalization factor.

\section{Charge $(Q-)$ Picture}

\subsection{Hidden Conformal Symmetry}

To probe the $Q$-picture $\mathrm{CFT}_{2}$ description one should impose $m=0$, and the the corresponding radial equation, from eq.(10), becomes

$$
\left(\partial_{r}\left(\Delta \partial_{r}\right)-\frac{\left[\left(r_{+}^{2}+a^{2}\right) \partial_{t}+\frac{Q r_{+}}{\ell} \partial_{\chi}\right]^{2}}{\left(r-r_{+}\right)\left(r_{+}-r_{-}\right)}+\frac{\left[\left(r_{-}^{2}+a^{2}\right) \partial_{t}+\frac{Q r_{-}}{\ell} \partial_{\chi}\right]^{2}}{\left(r-r_{-}\right)\left(r_{+}-r_{-}\right)}\right) \Phi=l(l+1) \Phi,
$$

where $\partial_{\chi}$ acts on "internal space" of the $U(1)$ symmetry such that $\partial_{\chi} \Phi=i \ell q \Phi$, and $\ell$ is a parameter depending on the embedding, see ${ }^{17,24}$ for detailed explanation. Similarly, the radial equation is just the Casimir operator of the $S L(2, R)$ Lie 
algebra $^{18}$ acting on $\Phi$ with the identifications

$$
\begin{aligned}
T_{L}^{Q} & =\frac{\left(r_{+}^{2}+r_{-}^{2}+2 a^{2}\right) \ell}{4 \pi Q\left(r_{+} r_{-}-a^{2}\right)}, & T_{R}^{Q} & =\frac{\left(r_{+}^{2}-r_{-}^{2}\right) \ell}{4 \pi Q\left(r_{+} r_{-}-a^{2}\right)}, \\
n_{L}^{Q} & =-\frac{r_{+}+r_{-}}{4\left(r_{+} r_{-}-a^{2}\right)}, & n_{R}^{Q} & =-\frac{r_{+}-r_{-}}{4\left(r_{+} r_{-}-a^{2}\right)} .
\end{aligned}
$$

Now since the momentum mode on the $\phi$ direction is turned off, such probe scalar field is not able to explore the information of the background rotation. Therefore, it can reveal only the charge dominated subsection resembling the $2 \mathrm{D}$ CFT dual to the RN black hole with

$$
c_{L}^{Q}=c_{R}^{Q}=\frac{6 Q^{3}}{\ell}
$$

and the CFT microscopic entropy matches the Bekenstein-Hawking area entropy of the KN black hole

$$
S_{\mathrm{CFT}}^{Q}=\frac{\pi^{2}}{3}\left(c_{L}^{Q} T_{L}^{Q}+c_{R}^{Q} T_{R}^{Q}\right)=\pi\left(r_{+}^{2}+a^{2}\right)=S_{\mathrm{BH}}
$$

\subsection{Scattering}

The solutions of the near region KG eq.(10), with $m=0$, are

$$
\begin{aligned}
R_{Q}^{(\text {in })} & =z^{-i \gamma_{Q}}(1-z)^{l+1} F\left(a_{Q}, b_{Q} ; c_{Q} ; z\right), \\
R_{Q}^{(\text {out })} & =z^{i \gamma_{Q}}(1-z)^{l+1} F\left(a_{Q}^{*}, b_{Q}^{*} ; c_{Q}^{*} ; z\right),
\end{aligned}
$$

where the expressions of $a_{Q}, b_{Q}, c_{Q}$ and $\gamma_{Q}$ are

$$
\begin{aligned}
& \gamma_{Q}=\frac{\omega\left(r_{+}^{2}+a^{2}\right)-q Q r_{+}}{r_{+}-r_{-}}, \quad a_{Q}=1+l-i \frac{\omega\left(r_{+}^{2}+r_{-}^{2}+2 a^{2}\right)-q Q\left(r_{+}+r_{-}\right)}{r_{+}-r_{-}}, \\
& b_{Q}=1+l-i\left[\omega\left(r_{+}+r_{-}\right)-q Q\right], \quad c_{Q}=1-i 2 \gamma_{Q} .
\end{aligned}
$$

The asymptotic solution of the ingoing mode is

$$
R_{Q}^{(\mathrm{in})}(r \gg M) \sim A_{Q} r^{l}+B_{Q} r^{-l-1},
$$

where

$$
A_{Q}=\frac{\Gamma\left(c_{Q}\right) \Gamma(2 l+1)}{\Gamma\left(a_{Q}\right) \Gamma\left(b_{Q}\right)}, \quad B_{Q}=\frac{\Gamma\left(c_{Q}\right) \Gamma(-2 l-1)}{\Gamma\left(c_{Q}-a_{Q}\right) \Gamma\left(c_{Q}-b_{Q}\right)}
$$

and

$$
h_{L}^{Q}=h_{R}^{Q}=l+1 .
$$

The coefficients $a_{Q}$ and $b_{Q}$ can be expressed as

$$
a_{Q}=h_{R}^{Q}-i \frac{\tilde{\omega}_{R}^{Q}}{2 \pi T_{R}^{Q}}, \quad b_{Q}=h_{L}^{Q}-i \frac{\tilde{\omega}_{L}^{Q}}{2 \pi T_{L}^{Q}},
$$

where

$$
\tilde{\omega}_{L}^{Q}=\omega_{L}^{Q}-q_{L}^{Q} \mu_{L}^{Q}, \quad \tilde{\omega}_{R}^{Q}=\omega_{R}^{Q}-q_{R}^{Q} \mu_{R}^{Q}
$$


with

$$
\begin{array}{ll}
\omega_{L}^{Q}=\frac{\ell \omega\left(r_{+}+r_{-}\right)\left(r_{+}^{2}+r_{-}^{2}+2 a^{2}\right)}{2 Q\left(r_{+} r_{-}-a^{2}\right)}, & q_{L}^{Q}=q, \quad \mu_{L}^{Q}=\frac{\ell\left(r_{+}^{2}+r_{-}^{2}+2 a^{2}\right)}{2\left(r_{+} r_{-}-a^{2}\right)} \\
\omega_{R}^{Q}=\frac{\ell \omega\left(r_{+}+r_{-}\right)\left(r_{+}^{2}+r_{-}^{2}+2 a^{2}\right)}{2 Q\left(r_{+} r_{-}-a^{2}\right)}, & q_{R}^{Q}=q, \quad \mu_{R}^{Q}=\frac{\ell\left(r_{+}+r_{-}\right)^{2}}{2\left(r_{+} r_{-}-a^{2}\right)} .
\end{array}
$$

The absorption cross section can be estimated by

$$
\left.P_{\mathrm{abs}}^{Q} \sim\left|A_{Q}\right|^{-2} \sim \sinh \left(2 \pi \gamma_{Q}\right)\right)\left|\Gamma\left(a_{Q}\right)\right|^{2}\left|\Gamma\left(b_{Q}\right)\right|^{2} .
$$

Again, with the help of the first law of thermodynamics $\delta S_{C F T}^{Q}=\delta S_{B H}$, the absorption cross section can be expressed as

$$
\begin{aligned}
P_{\mathrm{abs}}^{Q} \sim & \left(T_{L}^{Q}\right)^{2 h_{L}^{Q}-1}\left(T_{R}^{Q}\right)^{2 h_{R}^{Q}-1} \sinh \left(\frac{\tilde{\omega}_{L}^{Q}}{2 T_{L}^{Q}}+\frac{\tilde{\omega}_{R}^{Q}}{2 T_{R}^{Q}}\right) \\
& \times\left|\Gamma\left(h_{L}^{Q}+i \frac{\tilde{\omega}_{L}^{Q}}{2 \pi T_{L}^{Q}}\right)\right|^{2}\left|\Gamma\left(h_{R}^{Q}+i \frac{\tilde{\omega}_{R}^{Q}}{2 \pi T_{R}^{Q}}\right)\right|^{2},
\end{aligned}
$$

which matches the finite temperature absorption cross section of an operator with the conformal weights $\left(h_{L}, h_{R}\right)$, frequencies $\left(\omega_{L}, \omega_{R}\right)$ electric charges $\left(q_{L}, q_{R}\right)$ and chemical potentials $\left(\mu_{L}, \mu_{R}\right)$ in the dual $2 \mathrm{D}$ CFT with the temperatures $\left(T_{L}, T_{R}\right)$.

\subsection{Real-time Correlator}

In the $Q$-picture, the two-point retarded correlator is

$$
\begin{aligned}
G_{R}^{Q} \sim \frac{B_{Q}}{A_{Q}}= & (-)^{h_{L}^{Q}+h_{R}^{Q}} \sin \left(i \frac{\tilde{\omega}_{L}^{Q}}{2 T_{L}^{Q}}\right) \sin \left(i \frac{\tilde{\omega}_{R}^{Q}}{2 T_{R}^{Q}}\right) \\
& \times \Gamma\left(h_{L}^{Q}-i \frac{\tilde{\omega}_{L}^{Q}}{2 \pi T_{L}^{Q}}\right) \Gamma\left(h_{L}^{Q}+i \frac{\tilde{\omega}_{L}^{Q}}{2 \pi T_{L}^{Q}}\right) \\
& \times \Gamma\left(h_{R}^{Q}-i \frac{\tilde{\omega}_{R}^{Q}}{2 \pi T_{R}^{Q}}\right) \Gamma\left(h_{R}^{Q}+i \frac{\tilde{\omega}_{R}^{Q}}{2 \pi T_{R}^{Q}}\right) .
\end{aligned}
$$

At the Matsubara frequencies (28), the retarded Green function agrees well with the CFT results (26-27) up to a normalization factor depending on $q_{L}^{Q}$ and $q_{R}^{Q}$.

\section{Conclusion and Discussion}

In this short article, we briefly reviewed our recent work on the twofold dual 2D CFT descriptions of the generic nonextremal KN black hole by probing the hidden conformal symmetries, in terms of the Kerr/CFT and RN/CFT correspondences. The KN/CFTs dualities are shown to be supported by the matching of the entropies, absorption cross sections and real time correlators computed from both the gravity and the CFT sides. 
A geometric way to understand this multiple dual CFT pictures is that, the KN black hole contains two $U(1)$ fibers, one is the rotation another is the electromagnetic field. Its near extremal near horizon geometry has the isometry $S L(2, R)_{R} \times U(1) \times U(1)$, thus there are two options to form the desired warped $\mathrm{AdS}_{3}$ structures, consequently, leading two dual 2D CFT dualities. Therefore, besides the mass, each one of the other two macroscopic hairs of the KN black hole, can provide an individual holographically dual $\mathrm{CFT}_{2}$ description. This multiple dual CFT descriptions has been discussed for the higher dimensional rotating black holes with multiple rotations, in which each angular momentum can provide an individual dual $\mathrm{CFT}_{2}$, see for example ${ }^{26,30}$. It would be interesting to check our microscopic hair conjecture for more general black holes with multiple $U(1)$ charges, and find a full dual field theory description in which each CFT corresponds to some certain limit.

\section{Acknowledgement}

This work was supported by the National Science Council of the R.O.C. under the grant NSC 99-2112-M-008-005-MY3 and in part by the National Center of Theoretical Sciences (NCTS).

\section{References}

1. B. Bertotti, "Uniform electromagnetic field in the theory of general relativity," Phys. Rev. 116, 1331 (1959).

2. I. Robinson, "A Solution of the Maxwell-Einstein Equations," Bull. Acad. Pol. Sci. Ser. Sci. Math. Astron. Phys. 7, 351 (1959).

3. A. Strominger, C. Vafa, Phys. Lett. B379, 99-104 (1996). [hep-th/9601029].

4. G. 't Hooft, "Dimensional reduction in quantum gravity," arXiv:gr-qc/9310026.

5. L. Susskind, "The world as a hologram," J. Math. Phys. 36, 6377 (1995) [arXiv:hepth/9409089].

6. J. M. Maldacena, "The large N limit of superconformal field theories and supergravity," Adv. Theor. Math. Phys. 2, 231 (1998) [Int. J. Theor. Phys. 38, 1113 (1999)] [arXiv:hep-th/9711200].

7. S. S. Gubser, I. R. Klebanov and A. M. Polyakov, "Gauge theory correlators from non-critical string theory," Phys. Lett. B 428, 105 (1998) [arXiv:hep-th/9802109].

8. E. Witten, "Anti-de Sitter space and holography," Adv. Theor. Math. Phys. 2, 253 (1998) [arXiv:hep-th/9802150].

9. M. Guica, T. Hartman, W. Song and A. Strominger, "The Kerr/CFT correspondence," Phys. Rev. D 80, 124008 (2009) [arXiv:0809.4266 [hep-th]].

10. I. Bredberg, T. Hartman, W. Song and A. Strominger, "Black Hole Superradiance From Kerr/CFT," JHEP 1004, 019 (2010) [arXiv:0907.3477 [hep-th]].

11. A. Castro, A. Maloney and A. Strominger, "Hidden Conformal Symmetry of the Kerr Black Hole," arXiv:1004.0996 [hep-th].

12. C. M. Chen and J. R. Sun, "Hidden Conformal Symmetry of the Reissner-Nordstrom Black Holes," arXiv:1004.3963 [hep-th].

13. Y. Q. Wang and Y. X. Liu, "Hidden Conformal Symmetry of the Kerr-Newman Black Hole," arXiv:1004.4661 [hep-th].

14. B. Chen and J. Long, "Real-time Correlators and Hidden Conformal Symmetry in Kerr/CFT Correspondence," arXiv:1004.5039 [hep-th]. 
15. M. Becker, S. Cremonini and W. Schulgin, "Correlation Functions and Hidden Conformal Symmetry of Kerr Black Holes," arXiv:1005.3571 [hep-th].

16. B. Chen and J. Long, "On Holographic description of the Kerr-Newman-AdS-dS black holes," arXiv:1006.0157 [hep-th].

17. C. -M. Chen, Y. -M. Huang, J. -R. Sun, M. -F. Wu, S. -J. Zou, "On Holographic Dual of the Dyonic Reissner-Nordstrom Black Hole," Phys. Rev. D82, 066003 (2010). [arXiv:1006.4092 [hep-th]].

18. C. -M. Chen, Y. -M. Huang, J. -R. Sun, M. -F. Wu, S. -J. Zou, "Twofold Hidden Conformal Symmetries of the Kerr-Newman Black Hole," Phys. Rev. D82, 066004 (2010). [arXiv:1006.4097 [hep-th]].

19. Y. Matsuo, T. Tsukioka and C. M. Yoo, "Another Realization of Kerr/CFT Correspondence," Nucl. Phys. B 825, 231 (2010) [arXiv:0907.0303 [hep-th]].

20. A. Castro and F. Larsen, "Near Extremal Kerr Entropy from $A d S_{2}$ Quantum Gravity," JHEP 0912, 037 (2009) [arXiv:0908.1121 [hep-th]].

21. M. Cvetic and F. Larsen, "Greybody Factors and Charges in Kerr/CFT," JHEP 0909, 088 (2009) [arXiv:0908.1136 [hep-th]].

22. T. Hartman, K. Murata, T. Nishioka and A. Strominger, "CFT duals for extreme black holes," JHEP 0904, 019 (2009) [arXiv:0811.4393 [hep-th]].

23. M. R. Garousi and A. Ghodsi, "The RN/CFT Correspondence," Phys. Lett. B 687, 79 (2010) [arXiv:0902.4387 [hep-th]].

24. C. M. Chen, Y. M. Huang and S. J. Zou, "Holographic Duals of Near-extremal Reissner-Nordstrom Black Holes," JHEP 1003, 123 (2010) [arXiv:1001.2833 [hep-th]].

25. C. M. Chen, J. R. Sun and S. J. Zou, "The RN/CFT Correspondence Revisited," JHEP 1001, 057 (2010) [arXiv:0910.2076 [hep-th]].

26. H. Lu, J. Mei and C. N. Pope, "Kerr/CFT Correspondence in Diverse Dimensions," JHEP 0904, 054 (2009) [arXiv:0811.2225 [hep-th]].

27. D. D. K. Chow, M. Cvetic, H. Lu and C. N. Pope, "Extremal Black Hole/CFT Correspondence in (Gauged) Supergravities," Phys. Rev. D 79, 084018 (2009) [arXiv:0812.2918 [hep-th]].

28. H. Isono, T. S. Tai and W. Y. Wen, "Kerr/CFT correspondence and five-dimensional BMPV black holes," Int. J. Mod. Phys. A 24, 5659 (2009) [arXiv:0812.4440 [hep-th]].

29. X. N. Wu and Y. Tian, "Extremal Isolated Horizon/CFT Correspondence," Phys. Rev. D 80, 024014 (2009) [arXiv:0904.1554 [hep-th]].

30. C. M. Chen and J. E. Wang, "Holographic Duals of Black Holes in Five-dimensional Minimal Supergravity," Class. Quant. Grav. 27, 075004 (2010) [arXiv:0901.0538 [hepth]].

31. B. Chen and C. S. Chu, "Real-time correlators in Kerr/CFT correspondence," JHEP 1005, 004 (2010) [arXiv:1001.3208 [hep-th]].

32. M. Becker, S. Cremonini and W. Schulgin, "Extremal Three-point Correlators in Kerr/CFT," arXiv:1004.1174 [hep-th].

33. I. Bredberg, C. Keeler, V. Lysov and A. Strominger, "Cargese Lectures on the Kerr/CFT Correspondence," arXiv:1103.2355 [hep-th].

34. G. Compere, W. Song, A. Virmani, "Microscopics of Extremal Kerr from Spinning M5 Branes," [arXiv:1010.0685 [hep-th]].

35. D. T. Son and A. O. Starinets, "Minkowski-space correlators in AdS/CFT correspondence: Recipe and applications," JHEP 0209, 042 (2002) [arXiv:hep-th/0205051]. 